\title{
Prevalence Patterns of Chronic Kidney Disease among School Children and Adolescents in a School in Varanasi, India
}

\author{
Rai Pradeep K ${ }^{1 *}$, Rai Punam² and Bedi Sonam ${ }^{3}$ \\ ${ }^{1}$ Department of Nephrology, Opal Hospital, India \\ ${ }^{2}$ Senior Clinical Physiologist \& Infertility expert, Opal Hospital, India \\ ${ }^{3}$ Department of Medicine, Banaras Hindu University, India
}

\section{Research Article \\ Volume 4 Issue 1}

Received Date: January 09, 2019

Published Date: January 29, 2019

DoI: 10.23880 /oajun-16000154

*Corresponding author: Pradeep Kumar Rai, Senior Consultant Nephrologist, Department of Nephrology, Opal Hospital, Kakarmatta, Varanasi- 221004, India, Tel: +91-9336913486, Email: pradnephro@gmail.com; pradeepk.rai@gmail.com

\section{Abstract}

Background: Chronic kidney disease is relatively uncommon in children but can be a devastating illness with several long-term consequences. There is no consensus on the utility of screening programs of chronic kidney disease for children. Community based studies on prevalence of chronic kidney disease in children is very few. The present study aims to estimate the prevalence of chronic kidney disease in apparently healthy school children and adolescents in a screening program in a local school of Varanasi.

Methods: The study subjects constituted 118 healthy school children and adolescents, screened as a part of World Kidney Day celebration. Information on age, sex, height, weight, smoking and alcohol, history of diabetes mellitus and hypertension, familial history of kidney disease was extensively interrogated and clinical investigations like, urinalysis and serum creatinine levels were recorded. Chronic kidney disease outcome was detected based on estimated glomerular filtration rate levels and albuminuria. The association of each variable with the outcome was seen using Pearson's Chi square test.

Results: The overall prevalence of chronic kidney disease was 9.3\%. The median age of the study participants were 10 years. There was nearly equal proportion of children in both the sexes. Hypertension and albuminuria were associated with chronic kidney disease.

Conclusion: Children with chronic kidney disease comprise a very small but important portion of the total chronic kidney disease population. A regular screening process is required to study the prevalence of paediatric chronic kidney disease before one reaches to a stage of irreversible adverse outcome.

Keywords: Chronic kidney disease; Albuminuria; Children; Adolescents

Abbreviations: CKD: Chronic Kidney Disease, ESRD: End Stage Renal Disease, AKI: Acute Kidney Injury, DM: Diabetes Mellitus, HTN: Hypertension, SBP \& DBP:
Systolic and Diastolic Blood Pressures, BMI: Body Mass Index, RBS: Random Blood Sugar, eGFR: estimated Glomerular Filtration Rate, IQR: Inter Quartile Range, 
CAKUT: Congenital Anomalies of the Kidney and Urinary Tract, KDOQI: Kidney Disease Outcomes Quality Initiative, SPSS: Statistical Package for Social Sciences.

\section{Introduction}

The rising incidence of chronic kidney disease (CKD) is a major public-health problem [1]. Though relatively uncommon in children, it can be a devastating illness with several long-term consequences [2].

Epidemiologic data on CKD may underestimate its real incidence and prevalence since CKD is often clinically asymptomatic [3], especially in earlier stages. Existing evidences prove that end stage renal disease (ESRD) in children represents the 'tip of the iceberg' of CKD thus suggesting that the number of patients with earlier stages of the disease are likely to exceed those reaching ESRD. The estimates of CKD in majority of the studies had been based on the prevalence of moderate to severe renal disease and did not account the asymptomatic subjects [4].

Screening programs for early detection of chronic kidney disease are widely recommended [5-7]. However, there is no consensus on the utility of these screening programs for children. While, East-Asian countries have well-established urinary screening programs, western countries appear to be moving away from screening of asymptomatic children for early detection of CKD.

The incidence of paediatric CKD rose slowly during the 1980 s, then marginally until the first decade of the 21st century. The incidence of paediatric patients with end stage renal disease has increased almost 2 fold while the prevalence has increased 4 fold in the last 30 years [8]. Studies around the globe have reported the incidence of CKD in children to be between 3.0-12.1 cases per million; although the definition of CKD not being uniform in these studies with different thresholds of GFR, and the data were based on hospital records with varying age groups of children as well $[9,10]$. CKD prevalence in children had invariably varied worldwide in different settings be it in screening programs or in population or hospital based studies [11].

Community based studies on prevalence of CKD in children are very few. Hence, the present study aims to estimate the prevalence patterns of CKD in apparently healthy school children and adolescents in a screening program in Varanasi on World Kidney day, 2016.

\section{Methods}

The study subjects constituted apparently healthy children and adolescents from Apollo school, Varanasi. They were screened as a part of World Kidney Day celebration by Opal hospital on 10th March 2016. Considering 5\% prevalence of CKD in children, the sample size was estimated to be 115 children. A total of 118 children were considered in the present study. Children with age ranging between $3-18$ years were included in the study.

Approval to conduct this study was obtained from the Ethical and Research Committee of Opal Hospital, Varanasi. Informed consent was obtained from the children's parents or caregivers and school directors. Children with symptomatic acute kidney injury (AKI) or transplanted kidneys were excluded from the screening.

Information on socio-demographic profile and personal characteristics like, age, sex, height, weight, smoking and alcohol, history of diabetes mellitus (DM) and hypertension (HTN), familial history of kidney disease was extensively interrogated and clinical investigations like, urinalysis and serum creatinine levels were recorded. Systolic and diastolic blood pressures (SBP and DBP) were measured only on one occasion, i.e. on the day of screening.

Some of the variables were converted into categories for appropriate clinical interpretation. Age (in completed years) was divided into 4 intervals [12], e.g. toddler (ages 1-3 years); preschooler (ages 4-5 years); school-aged child (ages 6-11 years); adolescent (ages 12-18).

Gender was classified into two, boys and girls. Body mass index (BMI) was calculated in same way as adults using height and weight of children, but then compared against the percentile for children of the same sex and age for categorization [13].

Diabetes was defined as the use of glucose lowering medicine and/or, random blood sugar (RBS) $\geq 200$. Blood pressure was categorized into 3 groups: none, elevated BP and HTN, according to the published normative values [14]. Children were divided into two groups according to serum creatinine values: normal and abnormal based on paediatric reference values [15].

A urine dipstick (Medi-Test Combi 9-Macherey Nagel, Duren, Germany) was performed for each child for semi quantitative measurement of urine albumin [16]. Two milliliters of blood sample was collected from each 
participant through venipuncture to investigate for serum creatinine level. All subjects with a deranged renal function i.e. based on estimated glomerular filtration rate (eGFR) levels and presence of albuminuria were considered to be suffering with CKD. Furthermore, CKD stages were also categorized based on eGFR levels and albuminuria. GFR was calculated using the Schwartz equation for children and adolescents [17].

Albuminuria was regarded as significant if $1+$ and above [18]. CKD stages were defined as: stage 1 if eGFR $\geq 90$ and albuminuria present; stage 2 if eGFR $=60-89$ and albuminuria present; stage 3 if eGFR $=30-59$; stage 4 if eGFR= 15-29; and stage 5 if eGFR $<15$ according to the Kidney Disease Outcomes Quality Initiative (KDOQI) guidelines $[19,20]$. Children detected with CKD were referred to the paediatric nephrologist. No follow-up was done for them.

The association of each variable with the disease outcome, CKD, was seen separately using Pearson's Chi square test. All the analysis was performed on statistical package for social sciences (SPSS) version 18.

\section{Results}

The age-wise profile of the children and adolescents who participated in the study is presented in Table 1 \& Figure 1. The demographic and clinical profile of the study participants is given in Table 2. The median age (inter quartile range (IQR)) of the participants was $10(8-14)$ years. The overall prevalence of CKD was 9.3\% (11 of 118).

\begin{tabular}{|c|c|c|c|c|c|c|}
\hline \multicolumn{2}{|c|}{ Characteristics } & Overall & 1-3 years & 4-5 years & 6-11 years & 12-18 years \\
\cline { 2 - 7 } & N (\%) & N (\%) & N (\%) & N (\%) & N (\%) \\
\hline \multirow{3}{*}{ Gender } & Boys & $60(50.8)$ & $1(50.0)$ & $7(77.8)$ & $24(42.9)$ & $28(54.9)$ \\
\cline { 2 - 7 } & Girls & $58(49.2)$ & $1(50.0)$ & $2(22.2)$ & $32(57.1)$ & $23(45.1)$ \\
\hline \multirow{4}{*}{ Obesity } & Underweight & $5(4.2)$ & $0(0.0)$ & $1(11.1)$ & $4(7.1)$ & $0(0.0)$ \\
\cline { 2 - 7 } & Normal & $96(81.4)$ & $2(100.0)$ & $8(88.9)$ & $47(83.9)$ & $39(76.5)$ \\
\cline { 2 - 7 } & Overweight & $11(9.3)$ & $0(0.0)$ & $0(0.0)$ & $3(5.4)$ & $8(15.7)$ \\
\cline { 2 - 7 } & Obese & $6(5.1)$ & $0(0.0)$ & $0(0.0)$ & $2(3.6)$ & $4(7.8)$ \\
\hline \multirow{3}{*}{ BP problem } & No & $79(70.5)$ & $1(100.0)$ & $8(100.0)$ & $45(83.3)$ & $25(51.0)$ \\
\cline { 2 - 7 } & Elevated & $12(10.7)$ & $0(0.0)$ & $0(0.0)$ & $7(13.0)$ & $5(10.2)$ \\
\cline { 2 - 7 } Creatinine & Yes & $21(18.8)$ & $0(0.0)$ & $0(0.0)$ & $2(3.7)$ & $19(38.8)$ \\
\hline & Normal & $118(100.0)$ & $2(100.0)$ & $9(100.0)$ & $56(100.0)$ & $51(100.0)$ \\
\cline { 2 - 7 } eGFR & Abnormal & $0(0.0)$ & $0(0.0)$ & $0(0.0)$ & $0(0.0)$ & $0(0.0)$ \\
\hline & $\geq 90$ & $87(73.7)$ & $0(0.0)$ & $1(11.1)$ & $39(69.6)$ & $47(92.2)$ \\
\hline \multirow{2}{*}{ Albuminuria } & $60-89$ & $31(26.3)$ & $2(100.0)$ & $8(88.9)$ & $17(30.4)$ & $4(7.8)$ \\
\hline \multirow{2}{*}{ CKD } & Present & $11(9.3)$ & $0(0.0)$ & $0(0.0)$ & $4(7.1)$ & $7(13.7)$ \\
\cline { 2 - 7 } & Absent & $107(90.7)$ & $2(100.0)$ & $9(100.0)$ & $52(92.9)$ & $44(86.3)$ \\
\cline { 2 - 7 } & Present & $11(9.3)$ & $0(0.0)$ & $0(0.0)$ & $4(7.1)$ & $7(13.7)$ \\
\hline & Absent & $107(90.7)$ & $2(100.0)$ & $9(100.0)$ & $52(92.9)$ & $44(86.3)$ \\
\hline
\end{tabular}

Table 1: Age-wise profile of 118 screened school children.

\begin{tabular}{|c|c|c|c|c|}
\hline \multirow{2}{*}{ Characteristics } & \multirow{2}{*}{$\mathbf{N}$} & \multirow{2}{*}{$\%$ distribution } & \multirow{2}{*}{$\%$ CKD } & Pearson's $\chi^{2}$ \\
\hline & & & & p value \\
\hline \multicolumn{5}{|c|}{ Age (years) } \\
\hline 3-Jan & 2 & 1.7 & 0 & \multirow{4}{*}{0.455} \\
\hline 5-Apr & 9 & 7.6 & 0 & \\
\hline 11-Jun & 56 & 47.5 & 7.1 & \\
\hline 18-Dec & 51 & 43.2 & 13.7 & \\
\hline \multicolumn{5}{|c|}{ Gender } \\
\hline Male & 60 & 50.8 & 6.7 & 0.313 \\
\hline
\end{tabular}




\begin{tabular}{|c|c|c|c|c|}
\hline Female & 58 & 49.2 & 12.1 & \\
\hline \multicolumn{5}{|c|}{ Childhood obesity } \\
\hline Underweight & 5 & 4.2 & 0 & \multirow{4}{*}{0.826} \\
\hline Normal & 96 & 81.4 & 9.4 & \\
\hline Overweight & 11 & 9.3 & 9.1 & \\
\hline Obese & 6 & 5.1 & 16.7 & \\
\hline \multicolumn{5}{|c|}{ Blood pressure } \\
\hline No & 79 & 70.5 & 5.1 & \multirow{3}{*}{0.058} \\
\hline Elevated & 12 & 10.7 & 25 & \\
\hline HTN & 21 & 18.8 & 9.5 & \\
\hline \multicolumn{5}{|c|}{ Albuminuria } \\
\hline Present & 11 & 9.3 & 100 & \multirow{2}{*}{$<0.0001$} \\
\hline Absent & 101 & 90.7 & 0 & \\
\hline \multicolumn{5}{|c|}{ eGFR $\left(\mathrm{ml} / \mathrm{min} / 1.73 \mathrm{~m}^{2}\right)$} \\
\hline$\geq 90$ & 87 & 73.7 & 9.2 & \multirow{2}{*}{1} \\
\hline $60-89$ & 31 & 26.3 & 9.7 & \\
\hline
\end{tabular}

Table 2: Profile of participants and prevalence of CKD.

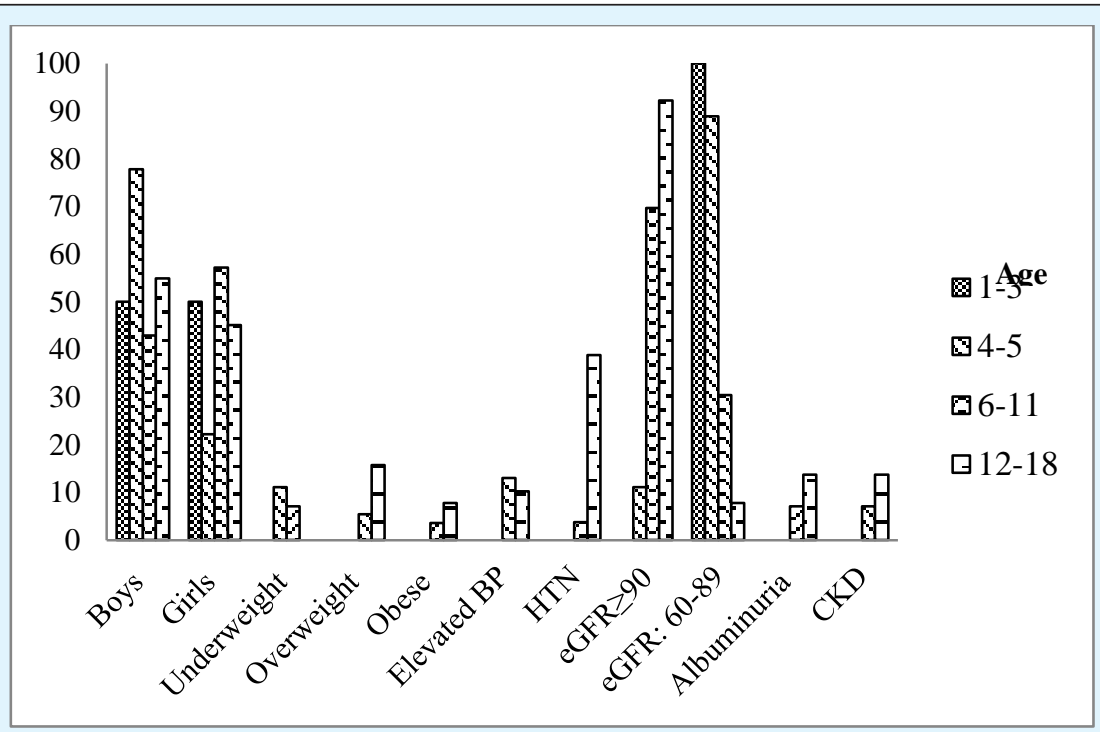

Figure 1: Age wise profile of children and adolescents.

There were almost equal number of subjects in the age groups 6-11 (47.5\%) and 12-18 (43.2\%) years, whereas only $9(7.6 \%)$ children were in the pre-school ages and only $2(1.7 \%)$ were toddlers. The prevalence of CKD was $7.1 \%$ among the school-aged children, and $13.7 \%$ among the adolescents $(p=0.455)$ whereas none in toddlers and pre-schoolers.

The study sample constituted nearly equal proportion of subjects from both the sexes $(50.8 \%$ males and $49.2 \%$ females). $6.7 \%$ boys and $12.1 \%$ girls were screened with CKD, but no statistical association was seen between CKD and gender $(p=0.313)$. None of the child was smoker/alcoholic. No one was found to be suffering with diabetes. The median (IQR) of RBS was 104 (95-113.25) $\mathrm{mg} / \mathrm{dl} .9 .3 \%$ and $5.1 \%$ subjects were overweight and obese with highest prevalence $(72.7 \%$ and $66.7 \%)$ among the adolescents. Only one participant each among the overweight and obese subjects had CKD $(9.1 \%$ and $16.7 \%$ respectively). Among normal BMI group, 9 (9.4\%) were 
screened with CKD. Almost equal number of boys (5\%) and girls $(5.2 \%)$ were suffering with childhood obesity, however, about one-third boys (36.4\%) and two-third girls $(63.6 \%)$ were overweight $(p=0.078)$. Childhood obesity was not associated with CKD occurrence $(\mathrm{p}=0.826)$.

None of the participant reported family history of renal disease. Elevated blood pressure and hypertension was found in nearly one-tenth (10.7\%) and one-fifth (18.8\%) subjects respectively. $25 \%$ children with elevated BP and 9.5\% with HTN were screened with CKD. The association was significant at $10 \%(\mathrm{p}=0.058)$.

The serum creatinine levels of all the subjects were within the normal range. Median serum creatinine level was $0.7(0.6-0.8) \mathrm{mg} / \mathrm{dl}$. Majority subjects (73.7\%) were falling in eGFR levels $\geq 90$ and only about one-fourth $(26.3 \%)$ in eGFR levels $60-89$ ( $p=1.000)$. Albuminuria was found positive in nearly one-tenth subjects $(9.3 \%)$ and was strongly associated with CKD $(\mathrm{p}<0.0001)$. The prevalence of children with eGFR $<75 \mathrm{~mL} / \mathrm{min} / 1.73 \mathrm{~m} 2$ was $1.7 \%$.

eGFR was strongly associated with age group of the children $(\mathrm{p}<0.0001)$; higher eGFR was seen in the adolescent and school age group (6-11 years) compared to lower eGFR levels in the pre-school children and toddlers. Out of all the hypertensive's, $90.5 \%$ were from the adolescent group and the result was highly significant ( $p<0.0001)$. Further, HTN was associated with presence of albuminuria and CKD outcome at 10\% ( $\mathrm{p}=0.058) .6 .8 \%$ children were in stage- 1 and $2.5 \%$ in stage- 2 CKD (Table $3)$.

\begin{tabular}{|c|c|c|c|c|}
\hline eGFR & $\mathbf{N}$ & \% distribution & Stages & \% with CKD staging \\
\hline$\geq 90$ & 87 & 73.7 & Stage- 1 & 6.8 \\
\hline $60-89$ & 31 & 26.3 & Stage- 2 & 2.5 \\
\hline
\end{tabular}

Table 3: CKD stages according to level of eGFR and albuminuria.

\section{Discussion}

The objective of the present study was to describe the prevalence patterns of CKD and its association with some known risk factors among apparently healthy school children and adolescents in a school in Varanasi.

The global prevalence of paediatric CKD through screening programs has varied from $<1-6 \%$, [21-24], although our study reported higher prevalence (9.3\%) compared to the published literature. However, a mass screening program in Korean school children with abnormal urinary findings depicted a CKD prevalence of $36.9 \%$ [25]. In the present study there were 6.8\% children in stage- 1 and $2.5 \%$ in stage- 2 CKD which was an under estimation of the previous published reports. The difference in the prevalence of CKD in various populations may be attributed to methodological differences used to define CKD, differences in cut-offs to define renal disease, geographic differences in the reporting of CKD and other renal disorders in children, due to environmental, racial, genetic, and cultural differences.

An abnormal eGFR in children and adolescents starts below $75 \mathrm{~mL} / \mathrm{min} / 1.73 \mathrm{~m} 2$, as a sign of renal disease. The prevalence of children in the present study with eGFR $<75$ $\mathrm{mL} / \mathrm{min} / 1.73 \mathrm{~m} 2$ was $1.7 \%$ which is consistent with the published literature [26].
Although CKD is more prevalent among boys in the previous studies [27], we did not find any gender differences with CKD in our study.

In our study, subjects with elevated BP had maximum (25\%) percentage of CKD, whereas 9.5\% hypertensive participants were suffering with CKD. Hypertension together with proteinuria has been shown to be an important risk factor for progression of primary renal disease in children and adults [28]. HTN has also been manifested responsible for deterioration of renal function in patients with CKD and their progression to higher stages of CKD. A similar study displayed $50 \%$ cases of chronic renal disease are hypertensive.

A screening program on World Kidney Day in Mexico yielded more systolic hypertension in younger children (19.6\% in age $0-8$ years; $7.1 \%$ in age $9-13$ years and $5.3 \%$ in age $14-17$ years) whereas in the present study, out of all the hypertensive, $90.5 \%$ were from the adolescent group and the association was highly significant. HTN was also associated with presence of albuminuria in our study which is a prologue to CKD occurrence and hence we found HTN association with CKD as well though not at 5\%. Cramer's V statistics depicted the strength of association of BP with albuminuria and eGFR was $22.5 \%$ and $23.6 \%$ respectively. 
Age is a factor strongly associated with the occurrence of CKD $[29,30]$. But the crucial difference between adult and paediatric ESRD lies in the aetiology of CKD. Adult CKD is predominately diabetic nephropathy and HTN whereas the top cause of paediatric CKD is congenital anomalies of the kidney and urinary tract (CAKUT), hereditary nephropathies and glomerulonephritis [31,32]. In the present research, age category was highly associated with blood pressure ( $\mathrm{p}<0.0001$ ). $90.5 \%$ and $41.7 \%$ adolescents and $9.5 \%$ and $58.3 \%$ school aged children were suffering with HTN and elevated BP respectively. Cramer's V statistics indicated the strength of association of blood pressure with age was $33.4 \%$.

Recent studies have identified obesity responsible for early kidney dysfunction and risk for CKD in children [33,34]. However our study reported no association between childhood obesity and CKD. The strong association of albuminuria with CKD, is in consistence with the fact that albuminuria is used as an important marker for CKD detection. eGFR was strongly associated with age groups of the children; higher eGFR was seen in the adolescent and school aged group (6-11 years) compared to lower eGFR levels in the pre-school children and toddlers. In children and adolescents, the increase in levels of creatinine with age and the differences in eGFR levels compared to adults are expected as creatinine production is related to muscle mass [35].

There were no subjects with diabetes in the present study however, there is an increasing prevalence of diabetes mellitus in children/adolescents across the globe [36] including India [37] in part with unfavorable lifestyle changes and reduced physical activity especially among youngsters provoking the rise in the prevalence of micro vascular complications like nephropathy over time.

The situation of asymptomatic CKD in the paediatric group is growing up. We screened apparently healthy school children and adolescents and found elevated BP and HTN in $10.7 \%$ and $18.8 \%$ respectively; which is potential risk factor for CKD. Moreover, CKD was detected in $>9 \%$ (11 of 118 ) subjects which is an alarming number. The findings of the present study cannot be generalized since they are based on the results of a single school only but gives the signal to emphasize on running screening programs at mass levels for detecting asymptomatic CKD in children and adolescents.

\section{Limitations}

The study subjects were screened in a cross sectional way on a single day and it was not possible to determine whether children who fulfilled outcome criteria did so for at least a 3-month period. Further, there was no follow up of the cases. Single day measurements of serum creatinine and urinary markers of kidney damage are likely to overestimate the true values. The study was conducted at a single school, though comprising students from all the sections of the society, but still there can be selection bias and the study results may not be generalized for the community based settings. Lastly, we did not do kidney ultrasounds, hence could have missed congenital anomalies of the kidney and urinary tract in children.

\section{Conclusion}

Children with CKD comprise a very small but important portion of the total CKD population. A regular screening process is required to study the prevalence of paediatric CKD and the potential impact of intervention if desired before one reaches to a stage of irreversible adverse outcome.

\section{Acknowledgements}

We would like to thank medical officers and staff of Opal hospital: Dr Shashi Kumar Gupta, Dr Hemant Kumar Rai, Dr Zafar Raja, Dr Surendra Nath Gupta, Mrs. Arti Maurya and Mr. Bansi Dhar Pandey; Principal, teachers and management staff of Apollo school and Yashwin Rai (MBBS student) who have helped with evaluation of subjects in the screening camp

\section{References}

1. Al-Kaabi A, Almaslamani T, Toaimah FH, Al-Maadid MG, Al-Bast DA, et al. (2011) Urinary screening for renal disease among apparently healthy school children in Qatar. J Trop Pediatr 58(1): 81-84.

2. Becherucci F, Roperto RM, Materassi M, Romagnani P (2016) Chronic kidney disease in children. Clin Kidney J 9(4): 583-591.

3. Soylemezoglu 0, Duzova A, Yalçınkaya F, Arınsoy T, Süleymanlar G (2012) Chronic renal disease in children aged 5-18 years: a population-based survey in Turkey, the CREDIT-C study. Nephrol Dial Transplant 27(3): 146-151.

4. Warady A, Chadha V (2007) Chronic kidney disease in children: the global perspective. Pediatr Nephrol 22(12): 1999-2009.

5. Rai PK, Rai P, Bedi S (2018) Prevalence and risk factors of chronic kidney disease: a single day screening on World kidney day for four consecutive 
years in Varanasi. Urol Nephrol Open Access J 6(5): 167-171.

6. Mendelssohn C (2005) Coping with the CKD epidemic: the promise of multidisciplinary teambased care. Nephrol Dial Transplant 20(1): 10-12.

7. Rai PK, Jindal PK, Rai P, Rai PK, Rai SN (2014) Screening of chronic kidney disease (CKD) in general population on world kidney day on three consecutive years: A single day data. Int J Med Public Heal 4(2): 167-170.

8. Baum M (2010) Overview of chronic kidney disease in children. Curr Opin Pediatr 22(2): 158-160.

9. Qader A, Uddin GM, Rahman H, Hanif M, Roy RR, et al. (2016) Renal Diseases in Children Attending Pediatric Nephrology Centers of Dhaka City. J Pediatr Nephrol 4(3): 86-91.

10. Sajid NK, Ahmad S, Sarwar I (2014) Chronic Renal Failure In Children; Etiology And Clinical Presentation. Prof Med J 21(2): 280-289.

11. Orantes-Navarro M, et al. (2016) Chronic kidney disease in children and adolescents in Salvadoran Farming Communities: NefroSalva Pediatric Study (2009-2011). MEDICC Rev 18(1-2): 15-21.

12. Kail RV (2011) Children and their Development. (Mydevelopmentlab Series), Engelwood Cliffs. NJ: Prentice Hall.

13. Wang Y, Chen HJ (2012) Use of percentiles and zscores in anthropometry in Handbook of anthropometry. Springer, 29-48.

14. Falkner B (2017) Changes in the 2017 pediatric hypertension clinical guidelines. Oxford University Press US.

15. Ghasemi A, Azimzadeh I, Afghan M, Momenan AA, Bagheripour F, et al. (2015) Pediatric reference values for serum creatinine and estimated glomerular filtration rate in Iranians: Tehran Lipid and Glucose Study. Arch Iran Med 18(11): 753-759.

16. Rai PK, Rai P, Bedi S (2018) Proteinuria as a Marker of Chronic Kidney Disease: A Single Centre Single Day Screening Experience for 9 Years in Varanasi. Open Access J Urol Nephrol 3(4): 1-8.
17. Schwartz GJ, Work DF (2009) Measurement and estimation of GFR in children and adolescents. Clin J Am Soc Nephrol 4(11): 1832-1843.

18. Ulasi II, Ijoma CK, Onodugo OD, Arodiwe EB, Ifebunandu NA, et al. (2013) Towards prevention of chronic kidney disease in Nigeria: a community-based study in Southeast Nigeria. Kidney Int Suppl 3(2): 195-201.

19. Ketteler M, Block GA, Evenepoel P, Fukagawa M, Herzog CA, et al. (2017) Executive summary of the 2017 KDIGO Chronic Kidney Disease-Mineral and Bone Disorder (CKD-MBD) Guideline Update: what's changed and why it matters. Kidney Int 92(1): 26-36.

20. Pottel H, Hoste L, Delanaye P (2015) Abnormal glomerular filtration rate in children, adolescents and young adults starts below $75 \mathrm{~mL} / \mathrm{min} / 1.73 \mathrm{~m}(2)$. Pediatr Nephrol 30(5): 821-828.

21. Fuhrman Y, Schneider MF, Dell KM, Blydt-Hansen TD, Mak R, et al. (2017) Albuminuria, proteinuria, and renal disease progression in children with CKD. Clin J Am Soc Nephrol 12(6): 912-920.

22. Lee Y, Baek S, Hong Kim J, Soo Kim D, Seung Lee J, et al. (2006) Analysis of renal biopsies performed in children with abnormal findings in urinary mass screening. Acta Paediatr 95(7): 849-853.

23. Tan SY, Naing L, Han A, Khalil MAM, Chong VH, et al. (2016) Chronic kidney disease in children and adolescents in Brunei Darussalam. World J Nephrol 5(2): 213-219.

24. Olowu WA, Adefehinti 0, Aladekomo TA (2013) Epidemiology and clinicopathologic outcome of pediatric chronic kidney disease in Nigeria, a single center study. Arab J Nephrol Transplant 6(2): 105113.

25. Cho BS, Kim SD, Choi YM, Kang HH (2001) School urinalysis screening in Korea: prevalence of chronic renal disease. Pediatr Nephrol 16(12): 1126-1128.

26. Koshy SM, Garcia-Garcia G, Pamplona JS, RenoirteLopez K, Perez-Cortes G, et al. (2009) Screening for kidney disease in children on World Kidney Day in Jalisco, Mexico. Pediatr Nephrol 24(6): 1219-1225.

27. Pounds LL, Teodorescu VJ (2013) Chronic kidney disease and dialysis access in women. J Vasc Surg 57(4): 49-53. 
28. Rodenbach E, Schneider MF, Furth SL, Moxey-Mims MM, Mitsnefes MM, et al. (2015) Hyperuricemia and progression of CKD in children and adolescents: the chronic kidney disease in children (CKiD) cohort study. Am J Kidney Dis 66(6): 984-992.

29. Baumgarten $M$, Gehr $T$ (2011) Chronic kidney disease: detection and evaluation. Am Fam Physician 84(10): 1138-1148.

30. Woodhouse S, Batten W, Hendrick H, Malek PA (2015) The glomerular filtration rate: an important test for diagnosis, staging, and treatment of chronic kidney disease. Lab Med 37(4): 244-246.

31. Harambat J, Van Stralen KJ, Kim JJ, Tizard EJ (2012) Epidemiology of chronic kidney disease in children. Pediatr Nephrol 27(3): 363-373.

32. Kaspar CD, Bholah R, Bunchman TE (2016) A review of pediatric chronic kidney disease. Blood Purif 41(13): 211-217.
33. Ding W, Cheung WW, Mak RH (2015) Impact of obesity on kidney function and blood pressure in children. World J Nephrol 4(2): 223-229.

34. Xiao N, Jenkins TM, Nehus E, Inge TH, Michalsky MP, et al. (2014) Kidney function in severely obese adolescents undergoing bariatric surgery. Obesity 22(11): 2319-2325.

35. Savory DJ (1990) Reference ranges for serum creatinine in infants, children and adolescents. Ann Clin Biochem 27(2): 99-101.

36. Rosenbloom AL, Silverstein JH, Amemiya S, Zeitler P, Klingensmith GJ (2009) Type 2 diabetes in children and adolescents. Pediatr Diabetes 10: 17-32.

37. Das AK (2015) Type 1 diabetes in India: Overall insights. Indian J Endocrinol Metab 19(1): 31-33. 